CONCRETE SHIELDING EXTERIOR TO IRON

P. Yurista and D. Cossairt

August 1983 


\section{CONCRETE SHIELDING EXTERIOR TO IRON}

P. Yurista, D. Cossairt

Many shielding situations require the efficient use of space. This frequently requires the use of high $\mathrm{Z}$ materials, particularly iron. A problem results in that iron does not efficiently attenuate neutrons below $1 \mathrm{Mev}^{(1)}$. Combining this with the dependence on energy for conversion to dose equivalent it is seen that special precautions need to be taken to optimize the use of high density of iron relative to concrete because of this leakage of low energy neutrons.

Reference 1 showed that at an equivalent star density the effect of the lower attenuation of neutrons less than 1 Mev by iron as compared to thick concrete results in a factor of $420 / 9.0=46.7$ increase in dose equivalent. To regain this lost factor of protection we need to provide additional attenuation for this specific portion of the spectrum. Hydrogenous material is the usual choice for shielding lower energy neutrons. Concrete because of its structural stability, low cost, and availability is used most frequently even though the water content is typically only $6 \%$. 
A "rule of thumb" at Fermilab has been to use 3 feet of concrete exterior to iron shielding. A recent design of a shield with a severe dimensional constraint has prompted a re-evaluation of this "rule of thumb" and has led to the following calculations of the concrete thickness required to nullify this problem.

The information used in these calculations include a neutron spectrum (fig. 1) due to o'Brien (2) at a depth of $2000 \mathrm{gm} / \mathrm{cm}^{2}$ in a $2000 \mathrm{gm} / \mathrm{cm}^{2}$ iron side-shielding of a particle accelerator. Protons lost during operation produce nuclear reactions or 'stars' upon deposition of their energy in the shielding material. These stars are the source of neutrons which make up the radiation field and the emitted spectrum is assumed to be independant of the proton energy initiating the nuclear reaction. The number of stars produced is proportional to the incident energy in GeV and the number of lost protons. Since we are concerned with relative magnitude and not an absolute magnitude these factors cancel out. The emergent spectrum from the shield is in $\mathrm{n} / \mathrm{cm}^{2}-\mathrm{sec}$. The conversion factors to mrem are from Patterson and Thomas (3) (fig. 2). These are dependant on the neutron energy and relate $\mathrm{n} / \mathrm{cm}^{2}-\mathrm{sec}$ to biological damage in mrem/hr. The dose-equivalent tenth-value layers in concrete are taken from NCRP $(1977)^{(4)}$ (fig. 3). A tenth-value layer 
is the thickness which is necessary to attenuate by a factor of ten. A concrete density of $2.4 \mathrm{gm} / \mathrm{cm}^{(3)}$ is used in conjunction with these values. The latter two sources are interpolated linearly where necessary.

The relative effect of concrete exterior to the iron on dose equivalent is the quantity sought. Using the spectrum of reference 2 we performed the following integrations for concrete thickness $x$ from $5 \mathrm{~cm}$ to $90 \mathrm{~cm}$ :

$$
\frac{\int \phi(E) K(E) 10^{-X / \lambda(E)} d E}{\int \phi(E) K(E) d E}
$$

Where $\phi, \mathrm{K}$ and $\lambda$ are the energy dependant functions of flux, dose equivalent conversion factor and tenth value layer respectively. The limits of integration were from 2.5E-8 Mev to 100 Mev although nearly all of the dose equivalent is expected from energies less than 1 Mev. The integration was conducted by two different methods as a check. One method was the ordinary trapezoidal rule and the other a cubic spline quadrature from an existing routine in the International Mathematical and Statistical Library (IMSL). There was no significant difference between the results. 
From a graph of the results (fig. 4) we see that to gain back our factor of 46.7 (attenuate by $1 / 46.7=.0214$ ) requires $60 \mathrm{~cm}$ of concrete exterior to the iron. For new construction this is a significant point in pouring a wall or floor to enclose iron shielding when compared to the previous 3 feet required. In the stacking of shield blocks it is less important due to the existing standard block sizes and stacking to preclude leakage through cracks. 


\section{REFERENCES}

1. P. Gollon, NAL TM 664 (1976).

2. K. O'Brien, HASL-240 "Neutron Spectra In the Side-Shielding of a Large Particle Accelerator" (1971).

3. H.W. Patterson and R.H. Thomas, Accelerator Health Physics, (Academic Press, New York, 1973), pg 69.

4. NCRP (1977), National Council on Radiation Protection and Measurements, Radiation Protection Design Guidelines for $0.1-100$ MeV Particle Accelerator Facilities, (National Council on Radiation Protection and Measurements, Washington), pg 123 . 


\section{FIGURES}

1. Neutron spectrum at $2000 \mathrm{gm} / \mathrm{cm}^{2}$ in $2000 \mathrm{gm} / \mathrm{cm}^{2} \mathrm{Fe}$ shield

2. Conversion factors from $\mathrm{n} / \mathrm{cm}^{2}-\mathrm{sec}$ to $\mathrm{mrem} / \mathrm{hr}$

3. Dose-Equivalent Tenth-value layers

4. Attenuation of dose equivalent vs concrete thickness exterior to iron. 


\section{Nevitionspecteum o $2000 \mathrm{gm} / \mathrm{cm}^{2}$}

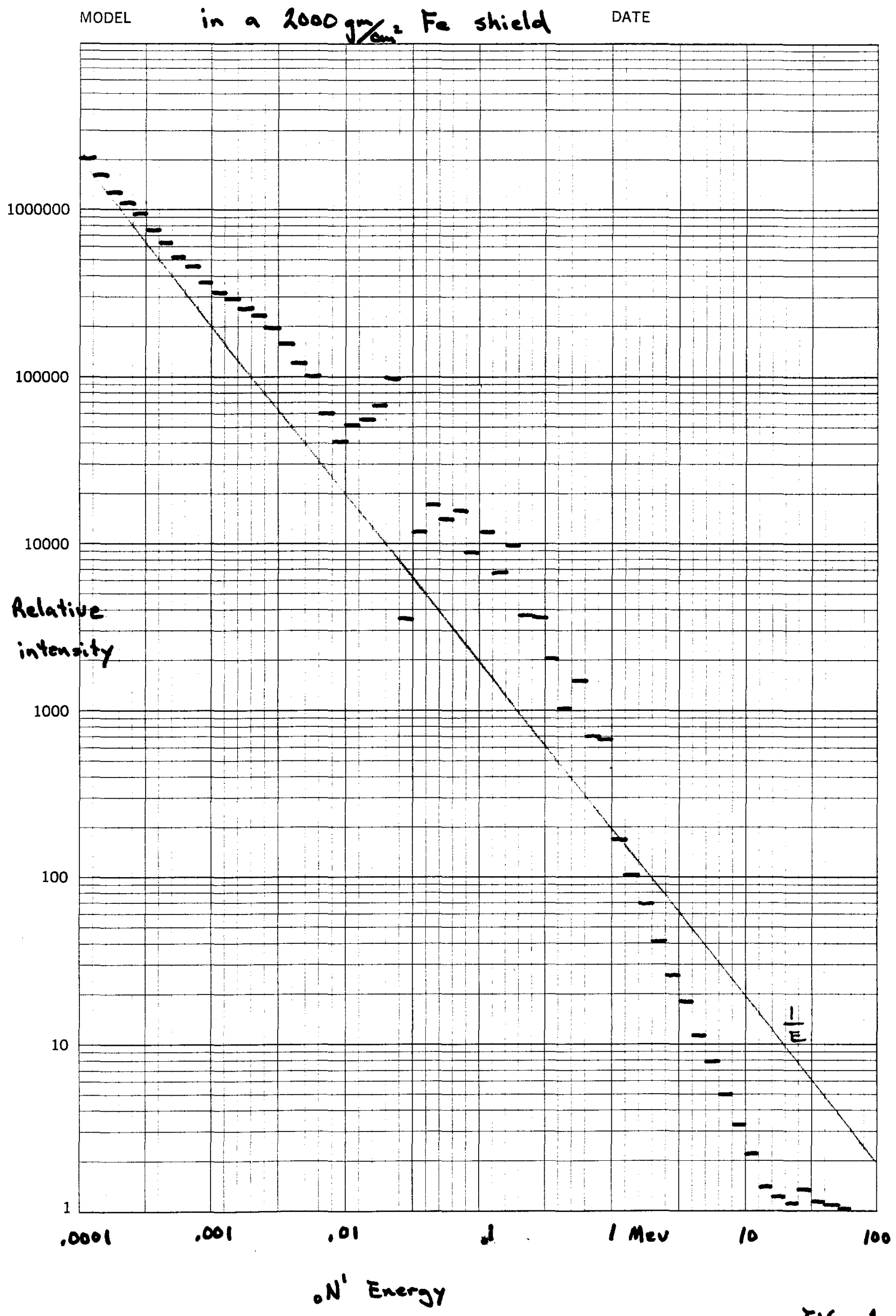

FIG. 1 


\section{F.10 Dose-Equivalent Index Tenth-Value Layers in Concrete for Monoenergetic Neutrons}

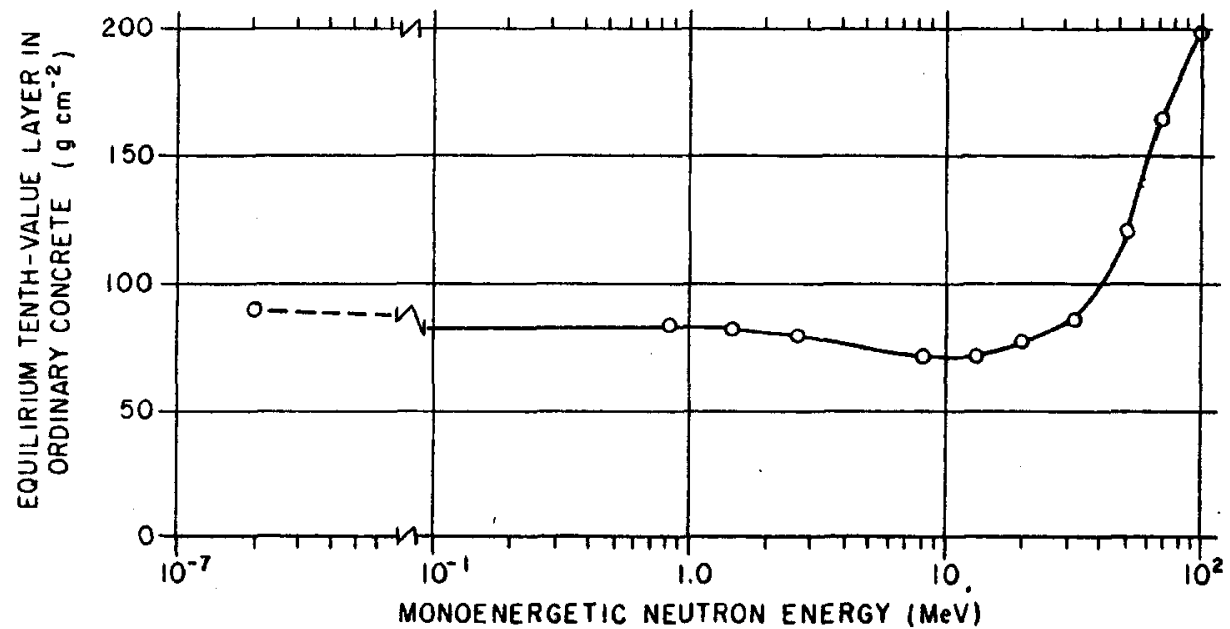

Equilibrium dose-equivalent index tenth-value layers, $T_{\mathrm{e}}$, for neutrons in ordinary concrete, as a function of monoenergetic-neutron energy. $T_{\mathrm{e}}$ is applicable only for transmission less than $10^{-16} \mathrm{rem} \mathrm{cm}^{2}$. The curve above is broken between $10^{-6}$ and $10^{-1} \mathrm{MeV}$ to indicate that the trend in $T_{\mathrm{c}}$ is very slow moving in thio energy range. Data were obtained directly from curves in Appendix F-6.

The equilibrium dose-equivalent index tenth-value layers for Appendices F-8 and F-9 are listed in Appendix F-7, accompanying the parameters of the corresponding neutron spectrum. Except for $(\gamma, n)$ spectra produced by electron energies greater than $55 \mathrm{MeV}$, and for neutrons produced by charged-particle energies greater than 20 $\mathrm{MeV}$, the values of $T_{\mathrm{e}}$ lie below $85 \mathrm{~g} \mathrm{~cm}^{-2}$.

Note: Refer to Section 4.3.3 regarding the limited usefulness of these data. 


\section{RADIATION FIELDS}

Table 2.IX. Conversion and modifying factors for neutrons.

(The number of significant digits given facilitates convenient numerical interpolation, and does not indicate the accuracy of the recommended factors.)

\begin{tabular}{|c|c|c|}
\hline $\begin{array}{l}\text { Neutron energy } \\
(\mathrm{MeV})\end{array}$ & $\begin{array}{r}\text { Conversion factor } \\
\left(\mathrm{n} / \mathrm{cm}^{2} \mathrm{sec} / \mathrm{mrem} / \mathrm{h}\right) \\
\end{array}$ & $\begin{array}{c}\text { Modifying } \\
\text { factor }\end{array}$ \\
\hline $2.5 \times 10^{-8}$ & 265 & 2.3 \\
\hline $5 \times 10^{-8}$ & 254 & 2.2 \\
\hline $1 \times 10^{-7}$ & 242 & 2.0 \\
\hline $2 \times 10^{-7}$ & 234 & 2.0 \\
\hline $5 \times 10^{-7}$ & 226 & 2.0 \\
\hline $1 \times 10^{-6}$ & 222 & 2.0 \\
\hline $2 \times 10^{-6}$ & 224 & 2.0 \\
\hline $5 \times 10^{-6}$ & 228 & 2.0 \\
\hline $1 \times 10^{-5}$ & 231 & 2.0 \\
\hline $2 \times 10^{-5}$ & 233 & 2.0 \\
\hline $5 \times 10^{-5}$ & 237 & 2.0 \\
\hline $1 \times 10^{-4}$ & 239 & 2.0 \\
\hline $2 \times 10^{-4}$ & 248 & 2.0 \\
\hline $5 \times 10^{-4}$ & 261 & 2.0 \\
\hline $1 \times 10^{-3}$ & 272 & 2.0 \\
\hline $2 \times 10^{-3}$ & 278 & 2.0 \\
\hline $5 \times 10^{-3}$ & 281 & 2.0 \\
\hline $1 \times 10^{-2}$ & 283 & 2.0 \\
\hline $2 \times 10^{-2}$ & 170 & 3.3 \\
\hline $5 \times 10^{-2}$ & 82 & 5.7 \\
\hline $1 \times 10^{-1}$ & 48 & 7.4 \\
\hline $2 \times 10^{-1}$ & 28 & 9.2 \\
\hline $5 \times 10^{-1}$ & 14 & 11.0 \\
\hline $1 \times 10^{0}$ & 8.5 & 10.6 \\
\hline $2 \times 10^{0}$ & 7.0 & 9.3 \\
\hline $5 \times 10^{0}$ & 6.8 & 7.8 \\
\hline $1 \times 10^{1}$ & 6.8 & 6.8 \\
\hline $2 \times 10^{1}$ & 6.5 & 6.0 \\
\hline $5 \times 10^{1}$ & 6.1 & 5.0 \\
\hline $1 \times 10^{2}$ & 5.55 & 4.4 \\
\hline $2 \times 10^{2}$ & 5.10 & 3.8 \\
\hline $5 \times 10^{2}$ & 3.60 & 3.2 \\
\hline $1 \times 10^{3}$ & 2.25 & 2.8 \\
\hline $2 \times 10^{3}$ & 1.55 & 2.6 \\
\hline
\end{tabular}

FIG. 3 


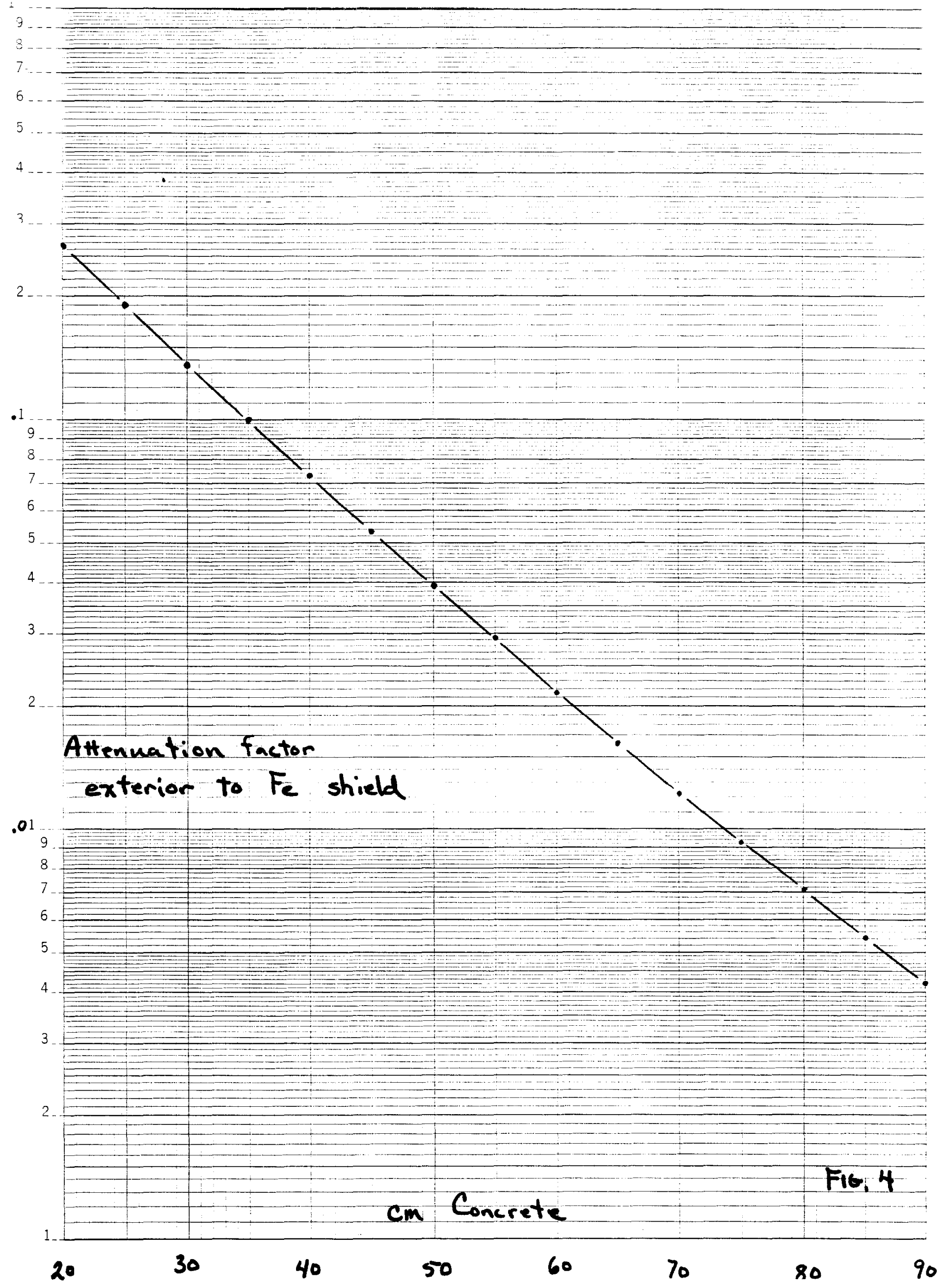

